\title{
The Effectiveness of Anticholinergic Therapy for Overactive Bladders: Systematic Review and Meta-Analysis
}

\section{Eficácia da terapia anticolinérgica na bexiga hiperativa: revisão sistemática e metanálise}

\author{
Andrea Moura Rodrigues Maciel da Fonseca ${ }^{1}$ Mariana Furtado Meinberg ${ }^{1}$ \\ Marilene Vale de Castro Monteiro ${ }^{1}$ Matheus Roque ${ }^{1}$ Jorge Milhen Haddad ${ }^{2}$ Rodrigo Aquino Castro ${ }^{3}$ \\ ${ }^{1}$ Urogynecology Division, Gynecology Discipline, Faculdade de \\ Medicina, Universidade Federal de Minas Gerais, Belo Horizonte, \\ MG, Brazil \\ Address for correspondence Andrea Moura Rodrigues Maciel da \\ Fonseca, PhD, Avenida Professor Alfredo Balena 190, 30130-100 - Belo \\ Horizonte, Minas Gerais, Brazil (e-mail: andreamrmf@gmail.com).
}

2 Urogynecology Division, Gynecology Discipline, Hospital das Clínicas,

Faculdade de Medicina, Universidade de São Paulo, São Paulo,

SP, Brazil

${ }^{3}$ Urogynecology Division, Gynecology Discipline, Escola Paulista de

Medicina, Universidade Federal de São Paulo, São Paulo, SP, Brazil

Rev Bras Ginecol Obstet 2016;38:564-575.

\begin{abstract}
Keywords

- overactive bladder

- urge incontinence

- urinary frequency and antimuscarinics
\end{abstract}

\section{Resumo}

The overactive bladder (OAB) has a significant negative impact on the quality of life of patients. Antimuscarinics have become the pharmacological treatment of choice for this condition. The objective of this systematic review and meta-analysis is to examine the evidence from randomized clinical trials about the outcomes of the antimuscarinic drugs available in Brazil on OABs. We searched MEDLINE and the Cochrane Central Register of Controlled Trials from the inception of these databases through to September 2015. The primary outcome measures were the mean decrease in urge urinary incontinence episodes and the mean decrease in the frequency of micturition. The results suggest that there is a moderate to high amount of evidence supporting the benefit of using anticholinergic drugs in alleviating $O A B$ symptoms when compared with placebo. It is still not clear whether any of the specific drugs that are available in Brazil offer advantages over the others. These drugs are associated with adverse effects (dry mouth and constipation), although they are not related to an increase in the number of withdrawals.

A bexiga hiperativa determina um impacto negativo na qualidade de vida dos nossos pacientes. Os antimuscarínicos tornaram-se o tratamento farmacológico de escolha para essa condição. O objetivo desta revisão sistemática e metanálise é examinar as melhores evidências científicas sobre estas medicações disponíveis no Brasil no tratamento de mulheres com bexiga hiperativa. As bases de dados utilizadas foram MEDLINE e a biblioteca da Cochrane, das quais selecionamos os ensaios clínicos received

November 27, 2015

accepted

September 30, 2016
DOI http://dx.doi.org/

10.1055/s-0036-1594289. ISSN 0100-7203.
Copyright $\odot 2016$ by Thieme-Revinter

Publicações Ltda, Rio de Janeiro, Brazil
License terms

(c) $(1) \$$ 
Palavras chaves

- bexiga hiperativa

- incontinência de urgência

- frequência urinária e antimuscarínicos randomizados até setembro de 2015. Os principais desfechos analisados foram a diminuição dos episódios de incontinência urinária de urgência e a diminuição da frequência de micção. Os resultados sugerem que as drogas existentes no Brasil sustentam o benefício dos anticolinérgicos no alívio dos sintomas da bexiga hiperativa quando comparadas com o placebo. Em termos de eficácia, as medicações apresentam resultados semelhantes no controle dos sintomas. Essas drogas estão associadas a efeitos adversos importantes, tais como boca seca e constipação, e esses efeitos adversos não influenciaram no uso da medicação.

\section{Introduction}

Overactive bladder $(\mathrm{OAB})$ is defined by the International Continence Society as the presence of urinary urgency, usually accompanied by frequency and nocturia, with or without urge urinary incontinence (UUI), in the absence of a urinary tract infection or another obvious pathology. ${ }^{1}$ Overactive bladder is a highly prevalent disease in both men and women, affecting $12-17 \%$ of the adult population. This condition has a significant negative impact on the quality of life of patients, affecting emotional, physical, social, occupational, and domestic functions. ${ }^{2-4}$

Overactive bladder symptoms are thought to develop as a result of inappropriate contractions of the bladder detrusor during the filling phase of the micturition cycle. Normal and abnormal bladder contractions occur via cholinergic activation of the muscarinic receptors. As is the case in other chronic conditions, $\mathrm{OAB}$ typically requires long-term persistence and adherence to therapy. ${ }^{5}$ Behavior modification, which includes education about the disorder, lifestyle changes (, such as avoiding caffeinated beverages, for example), as well as pelvic floor muscle training and bladder retraining, represent the first-line therapy options for this condition. However, when these approaches are insufficient, second-line therapy involves pharmacological treatment, and antimuscarinic agents are the treatment of choice. ${ }^{6-8}$

Although anticholinergic medications have been shown to improve patients' symptoms, they create a widespread blockade of cholinergic activity that often results in side effects such as dry mouth, cognitive changes, constipation, urinary retention, blurred vision, and dyspepsia. ${ }^{9}$ These problems can be difficult to manage, and may contribute to poor patient adherence to treatment. ${ }^{10}$

The objective of this systematic review and meta-analysis was to examine the currently available evidence from randomized clinical trials (RCTs) about the outcomes of the pharmacological management of $\mathrm{OAB}$, and to summarize the comparative effectiveness of the drugs available in Brazil. Only antimuscarinic agents commercialized in Brazil were included in the analysis, since this meta-analysis is the basis for the development of Brazilian urogynecology guidelines. ${ }^{11}$

\section{Methods}

This study was exempt from institutional review board approval, given that it was a systematic review and meta- analysis; it did not involve the use of any interventions on humans. To report the results of this meta-analysis, we utilized the Preferred Reporting Items for Systematic reviews and Meta-Analysis (PRISMA) statement. ${ }^{12}$

\section{Search Strategy}

An exhaustive electronic search was performed using the MEDLINE database, as well as the Cochrane Central Register of Controlled Trials, with the dates of the included articles spanning from the inception of these databases through to September 2015. We also searched the references of the identified articles and restricted the search to articles published in English. The search combined relevant terms and descriptors related to $\mathrm{OAB}$, anticholinergic drugs, oxybutynin, darifenacin, tolterodine, solifenacin, and RCTs.

\section{Eligibility Criteria and Data Extraction}

The review only included RCTs featuring adult male and female patients diagnosed with OAB or with a diagnosis of detrusor over activity, and who were also submitted to any of the anticholinergic treatments available in Brazil. The selection criteria are described in - Table 1. In a first screening, two independent authors (AMRMF and MVCM) assessed all of the abstracts retrieved from the search; they then obtained the full manuscripts of the citations that met the inclusion criteria. These authors evaluated the studies' eligibility and quality, and extracted the data subsequently. Any discrepancies were solved by agreement, and, if needed, the authors reached a consensus with a third author (MR). The meta-analysis included studies that provided accurate data related to those primary outcomes that could be analyzed. Thus, only studies that provided the mean, sample size, and standard deviation (SD) values of the primary outcomes were included in the analysis. Otherwise, when the available data were expressed as the median, it was necessary that the study provided the range values (lowest and highest values) to extrapolate the mean. If only the ranges of continuous variables were reported, we would estimate the SD by dividing the range by four. Dose escalation and crossover studies were excluded, as it was not possible to abstract the data related to our primary outcomes.

\section{Outcome Measures}

The primary outcomes of interest for this systematic review and meta-analysis were the mean decrease in the number of UUI episodes per day and the mean decrease in the number 
Table 1 Selection criteria of included studies (PICOs)

\begin{tabular}{|c|c|c|}
\hline & Included & Excluded \\
\hline Population & $\begin{array}{l}\text { Symptomatic diagnosis of overactive bladder (OAB) or } \\
\text { a urodynamic diagnosis of detrusor over activity }\end{array}$ & $\mathrm{OAB}$ as consequence of surgery \\
\hline Intervention & $\begin{array}{l}\text { Anticholinergic drugs available in Brazil (oxybutynin } \\
5 \mathrm{mg} \text { and } 10 \mathrm{mg} \text {; darifenacin } 7.5 \mathrm{mg} \text { and } 15 \mathrm{mg} \text {; } \\
\text { tolterodine } 1 \mathrm{mg}, 2 \mathrm{mg} \text {, and } 4 \mathrm{mg} \text {; solifenacin } 5 \mathrm{mg} \\
\text { and } 10 \mathrm{mg} \text { ) }\end{array}$ & $\begin{array}{l}\text { Anticholinergic drugs not available in Brazil or with } \\
\text { different doses or routes of administration that are not } \\
\text { available in Brazil }\end{array}$ \\
\hline Comparison & $\begin{array}{l}\text { Placebo, comparison between different drugs, or } \\
\text { comparison between different doses of the same drug }\end{array}$ & \\
\hline Outcomes & $\begin{array}{l}\text { Primary outcomes } \\
\text { - Mean decrease in urge urinary incontinence (UUI) } \\
\text { episodes per day } \\
\text { - Mean decrease in the number of micturitions per } \\
\text { day } \\
\text { Secondary outcomes } \\
\text { - Mean decrease in total incontinence episodes } \\
\text { (related or not to urgency) } \\
\text { - Dry mouth } \\
\text { - Constipation } \\
\text { - Withdrawals resulting from drug-related adverse } \\
\text { effects }\end{array}$ & \\
\hline Study type & Randomized controlled trials (RCTs) & Non RCTs \\
\hline
\end{tabular}

Abbreviation: PICOs, population, intervention, comparison and outcomes.

of micturitions per day. The secondary outcomes included the mean decrease in total incontinence episodes (either related or not to urgency), dry mouth, constipation, and withdrawals resulting from drug-related adverse effects. We tried to perform meta-analytic comparisons between each drug (and their different dosages) versus placebo, comparisons between different drugs, and comparisons between different dosages of the same drug.

\section{Risk of Bias Assessment}

We followed the guidance suggested by the Cochrane Collaboration $^{13}$ to assess the risk of bias from the included studies. We evaluated sequence generation, allocation concealment, blinding, and incomplete outcome data for each trial included in the review. A low risk of bias was considered when a judgment of "yes" for all domains was obtained, whereas a high risk of bias was considered when a judgment of "no" for one or more domains was obtained. An unclear risk of bias was defined when an "unclear" judgment in any domain was considered. The quality assessment of the included trials is shown in - Table 2 .

\section{Analysis}

We pooled the data of the continuous outcomes from the original studies to obtain the mean difference (MD) for the occurrence of an outcome event, and presented their corresponding 95\% confidence intervals (CIs). Data for dichotomous outcomes from the original studies were pooled to obtain the relative ratio (RR), and the corresponding 95\% CIs were calculated. Statistical significance was set at a $p$-value of $<0.05$. In order to quantify the statistical heterogeneity, we used the I 2 statistic to describe the variations across trials that were due to heterogeneity and not to sampling error. We pooled the outcome data from each study using a MantelHaenszel model, and applied the fixed-effects model. When the heterogeneity was greater than $50 \%$ (I2 > 50\%), we applied the random-effects model. ${ }^{14}$ We used the software Review Manager (RevMan, Version 5.3; Copenhagen) to conduct the meta-analysis.

\section{Results}

Our electronic search retrieved 468 articles. After screening the titles and abstracts, we ended up with 37 articles that were considered eligible for inclusion in this review by one or both reviewers, and the full texts were subsequently assessed. The complete article selection process is presented in -Fig. 1.

\section{Description of Included Studies}

Fifteen RCTs assessing the pharmacological management (drugs and dosages available in Brazil) of OAB met the inclusion criteria and provided data to perform the metaanalysis. With the available data of the included studies, ${ }^{15-29}$ it was only possible to perform comparisons between tolterodine (and its different dosages) versus placebo, solifenacin versus placebo, and oxybutynin versus tolterodine.

\section{Excluded Studies}

Twenty-two articles were excluded because they either did not meet the inclusion criteria ${ }^{30-35}$ or they did not provide adequate data to be included in the meta-analysis. ${ }^{36-51}$ 
Effectiveness of Anticholinergic Therapy for Overactive Bladders Fonseca et al. 567

Table 2 Quality assessment of included trials

\begin{tabular}{|c|c|c|c|c|}
\hline Study & Sequence generation & Allocation concealed & Blinding & Incomplete outcome data \\
\hline Appell et al. ${ }^{15}$ & Unclear & Yes & Yes & Yes \\
\hline Drutz et al. $^{16}$ & Unclear & Yes & Yes & Yes \\
\hline Lee et al. ${ }^{17}$ & Yes & Yes & Yes & Yes \\
\hline Malone-Lee et al. ${ }^{18}$ & Unclear & Unclear & Yes & Yes \\
\hline Chapple et al. ${ }^{19}$ & Unclear & Yes & Unclear & Yes \\
\hline Jacquetin et al. ${ }^{20}$ & Unclear & Yes & Unclear & Yes \\
\hline Khullar et al. ${ }^{21}$ & Yes & Yes & Yes & Yes \\
\hline Millard et al. ${ }^{22}$ & Unclear & Unclear & Unclear & Yes \\
\hline Swift et al. ${ }^{23}$ & Yes & Yes & Yes & Yes \\
\hline Van Kerrebroeck et al. ${ }^{24}$ & Unclear & Unclear & Unclear & Yes \\
\hline Van Kerrebroeck et al. ${ }^{25}$ & Yes & Yes & Yes & Yes \\
\hline Zinner et al. ${ }^{26}$ & Yes & Yes & Yes & Yes \\
\hline Cardozo et al. ${ }^{27}$ & Unclear & Unclear & Unclear & Yes \\
\hline Karram et al. ${ }^{28}$ & Unclear & Unclear & Unclear & Yes \\
\hline But et al. ${ }^{29}$ & Yes & Unclear & Unclear & Yes \\
\hline
\end{tabular}
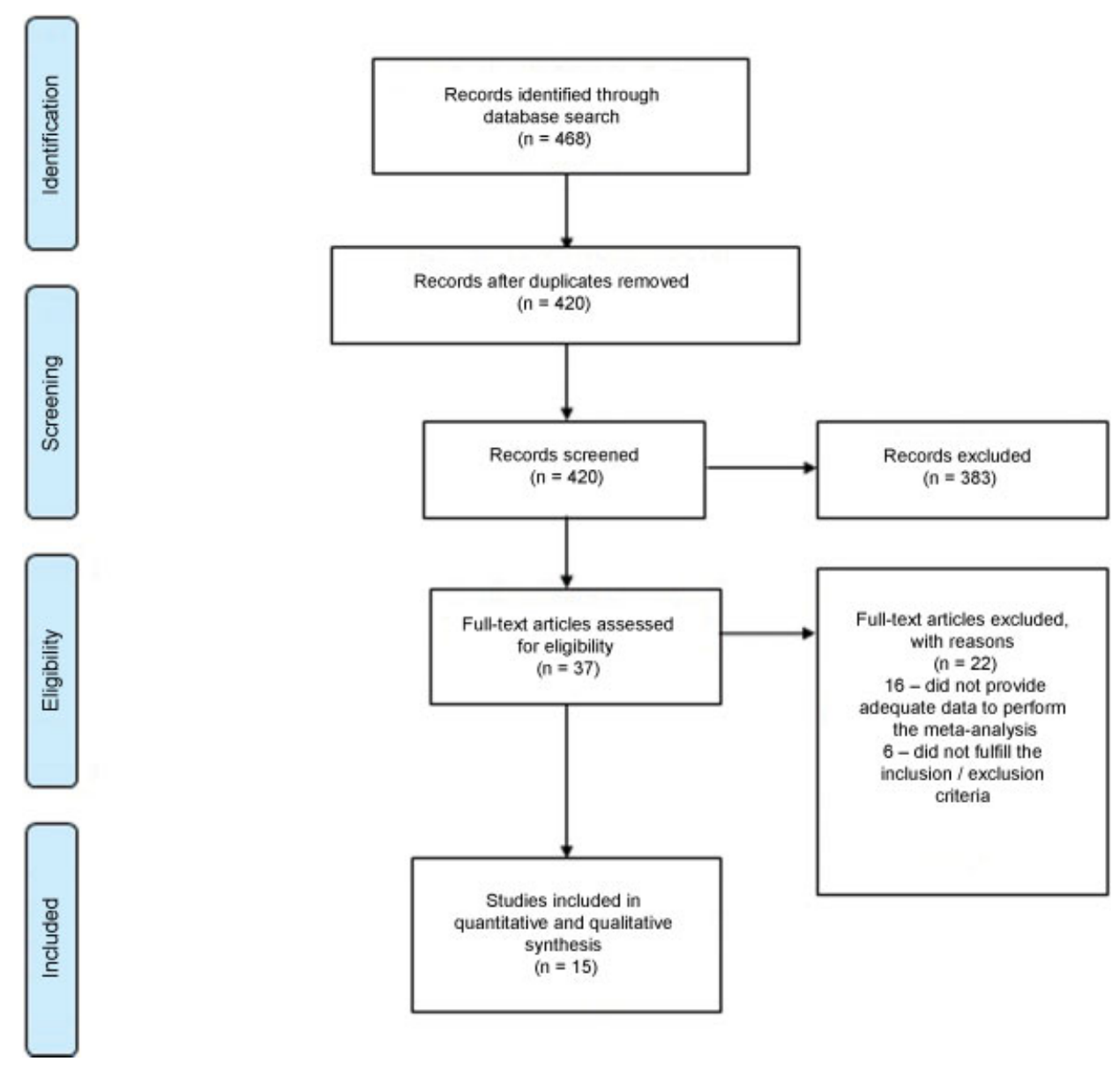

Fig. 1 Flowchart for the trial identification and selection process. 


\section{Oxybutynin vs. Tolterodine}

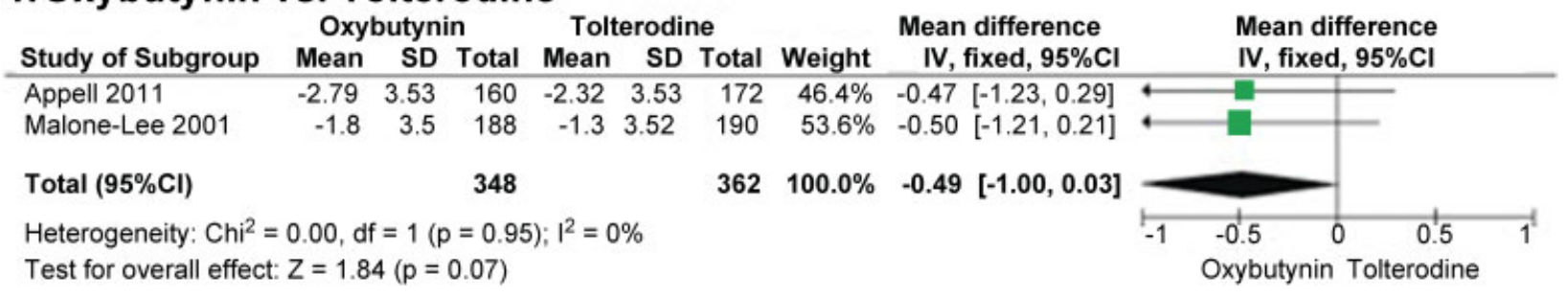

Fig. 2 Forest plot - mean difference in decrease in urge urinary incontinence (UUI) episodes per day.

\section{Primary Outcomes}

- Mean decrease in UUI episodes per day

For this outcome, it was only possible to perform a comparison between oxybutynin and tolterodine. The MD in the mean decrease in UUI episodes per day was higher for patients that used oxybutynin than for those that used tolterodine $(\mathrm{MD}=-0.49 ; 95 \% \mathrm{Cl}:-1.00,0.03 ; \mathrm{I} 2=0$; $p=0.07$ ); however, this difference was not significant (-Fig. 2).

- Mean decrease in the number of micturitions per day

We were able to perform the following comparisons: oxybutynin versus tolterodine; tolterodine (and its different doses) versus placebo; different doses of tolterodine; and solifenacin versus placebo. We found significant differences that favored tolterodine $1 \mathrm{mg}$ when compared with placebo $(\mathrm{MD}=-0.55$; 95\% CI: $-1.08,-0.02 ; \mathrm{I} 2=0 ; p=0.04)$; tolterodine $2 \mathrm{mg}$ versus placebo (MD $=-0.57 ; 95 \% \mathrm{CI}:-0.82,-0.32 ; \mathrm{I} 2=0 ; p<0.001$ ); and tolterodine $4 \mathrm{mg}$ versus placebo $(\mathrm{MD}=-0.66 ; 95 \% \mathrm{Cl}$ : $0.85,-0.47 ; \mathrm{I} 2=0 ; p<0.001)$. Moreover, significant differences favored the use of solifenacin when compared with placebo $(\mathrm{MD}=-0.77 ; 95 \% \mathrm{Cl}:-1.09,-0.45 ; \mathrm{I} 2=0 ; p<0.001)$ (-Fig. 3 ). All of these outcome data were pooled from each study using a Mantel-Haenszel model, and a fixed-effects model was applied, as there was no heterogeneity ( $\mathrm{I} 2=0$ ) among the studies. For all other available comparisons for this outcome (oxybutynin versus tolterodine; tolterodine $2 \mathrm{mg}$ versus tolterodine $1 \mathrm{mg}$; and tolterodine $4 \mathrm{mg}$ versus tolterodine $2 \mathrm{mg}$ ), the MD was not significant, as presented in - Fig. $\mathbf{3}$.

\section{Secondary Outcomes}

- Mean decrease in incontinence episodes per day

Significant differences were found that favored tolterodine $2 \mathrm{mg}$ when compared with placebo (MD $=-0.45 ; 95 \% \mathrm{Cl}$ : $0.76,-0.14 ; \mathrm{I} 2=0 ; p=0.005)$; tolterodine $4 \mathrm{mg}$ versus placebo ( $\mathrm{MD}=-0.46$; $95 \% \mathrm{CI}$ : $-0.83,-0.08$; I2 $=0 ; p=0.02$ ); and solifenacin versus placebo ( $\mathrm{MD}=-0.77 ; 95 \% \mathrm{CI}:-1.09,-0.45$; $\mathrm{I} 2=0 ; p<0.001$ ) ( - Fig. 4). All of these outcome data were pooled from each study using a Mantel-Haenszel model, and a fixed-effects model was applied, as there was no heterogeneity $(\mathrm{I} 2=0)$ among the studies. We did not find significant differences across any of the other available comparisons (oxybutynin versus tolterodine; tolterodine $1 \mathrm{mg}$ versus placebo; tolterodine $2 \mathrm{mg}$ versus tolterodine $1 \mathrm{mg}$; and tolterodine $4 \mathrm{mg}$ versus tolterodine $2 \mathrm{mg}$ ).

\section{- Dry mouth}

There were significant differences and higher RRs in patients treated with oxybutynin when compared with tolterodine $(\mathrm{RR}=1.49 ; 95 \% \mathrm{CI}: 1.06,2.10 ; \mathrm{I} 2=84 \% ; p=0.02)$; tolterodine $1 \mathrm{mg}$ versus placebo $(\mathrm{RR}=2.33$; $95 \% \mathrm{CI}$ : $1.26,4.29$; $\mathrm{I} 2=84 \%$; $p=0.02)$; tolterodine $2 \mathrm{mg}$ versus placebo $(\mathrm{RR}=3.72 ; 95 \% \mathrm{CI}$ : $3.05,4.54 ; \mathrm{I} 2=0 \% ; p<0.001$ ); tolterodine $4 \mathrm{mg}$ versus placebo $(\mathrm{RR}=2.88 ; 95 \% \mathrm{CI}: 2.40,3.45 ; \mathrm{I} 2=0 \% ; p<0.001)$; tolterodine $2 \mathrm{mg}$ versus tolterodine $1 \mathrm{mg}(\mathrm{RR}=1.69 ; 95 \% \mathrm{CI}$ : $1.26,2.28$; $\mathrm{I} 2=0 \% ; p<0.001)$, and solifenacin versus placebo $(\mathrm{RR}=3.73$; $95 \% \mathrm{CI}: 1.80,7.77 ; \mathrm{I} 2=0 \% ; p<0.001$ ). The group of patients that used tolterodine $4 \mathrm{mg}$ exhibited a lower risk $(\mathrm{RR}=0.79 ; 95 \% \mathrm{CI}$ : $0.68,0.92 ; \mathrm{I} 2=0 \% ; p=0.02$ ) when compared with tolterodine $2 \mathrm{mg}$. All of these results are presented in -Fig. 5 .

\section{- Constipation}

The findings indicated that there was a significant difference and a higher RR in patients treated with tolterodine $2 \mathrm{mg}$ versus those treated with placebo $(\mathrm{RR}=1.61 ; 95 \% \mathrm{CI}$ : $1.11,2.32 ; \mathrm{I} 2=0 \% ; p=0.01$ ), and those treated with tolterodine $4 \mathrm{mg}$ versus placebo $(\mathrm{RR}=1.52 ; 95 \% \mathrm{CI}$ : $1.11,2.09$; $\mathrm{I} 2=0 \% ; p=0.009$ ). We did not find significant differences across any of the other available comparisons (oxybutynin versus tolterodine; solifenacin versus placebo). All of these results are presented in -Fig. 6 .

- Withdrawals resulting from drug-related adverse effects

We did not find statistical differences in any of the available comparisons (oxybutynin versus tolterodine, $p=0.18$; tolterodine $1 \mathrm{mg}$ versus placebo, $p=0.47$; tolterodine $2 \mathrm{mg}$ versus placebo, $p=0.32$; tolterodine $4 \mathrm{mg}$ versus placebo, $p=0.13$; tolterodine $2 \mathrm{mg}$ versus tolterodine $1 \mathrm{mg}, p=0.59$; tolterodine $4 \mathrm{mg}$ versus tolterodine $2 \mathrm{mg}, p=0.92$; and solifenacin versus placebo, $p=0.67$ ) when evaluating the risk of withdrawals due to drug-related adverse effects.

\section{Discussion}

To our knowledge, this is the first comprehensive review featuring a pooled analysis that has addressed the question of efficacy and the main adverse effects of all antimuscarinic drugs available in Brazil for the treatment of OAB. 
1. Oxybutynin vs. Tolterodine

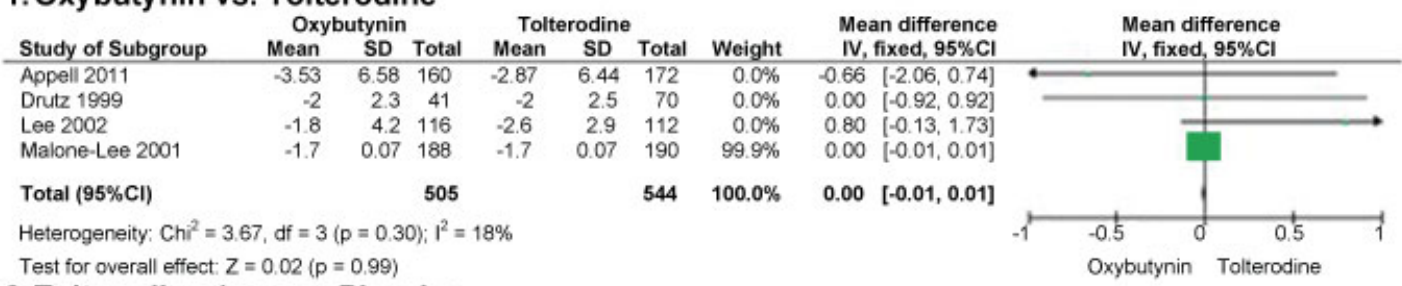

2. Tolterodine $1 \mathrm{mg}$ vs. Placebo

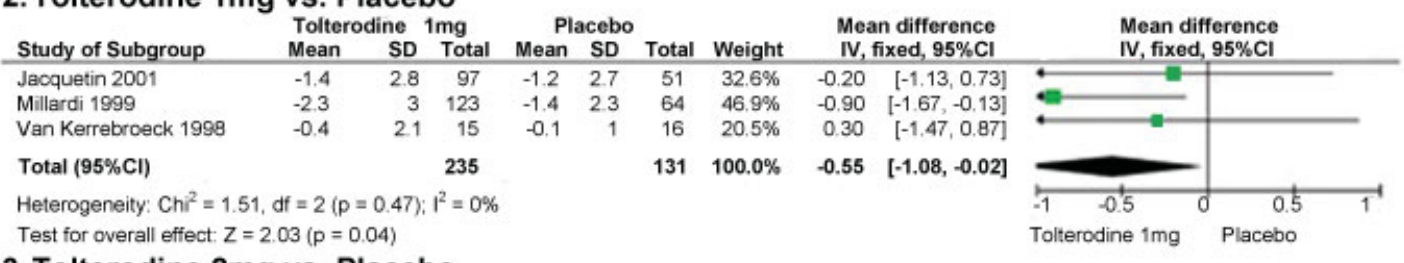

\section{Tolterodine $2 \mathrm{mg}$ vs. Placebo}

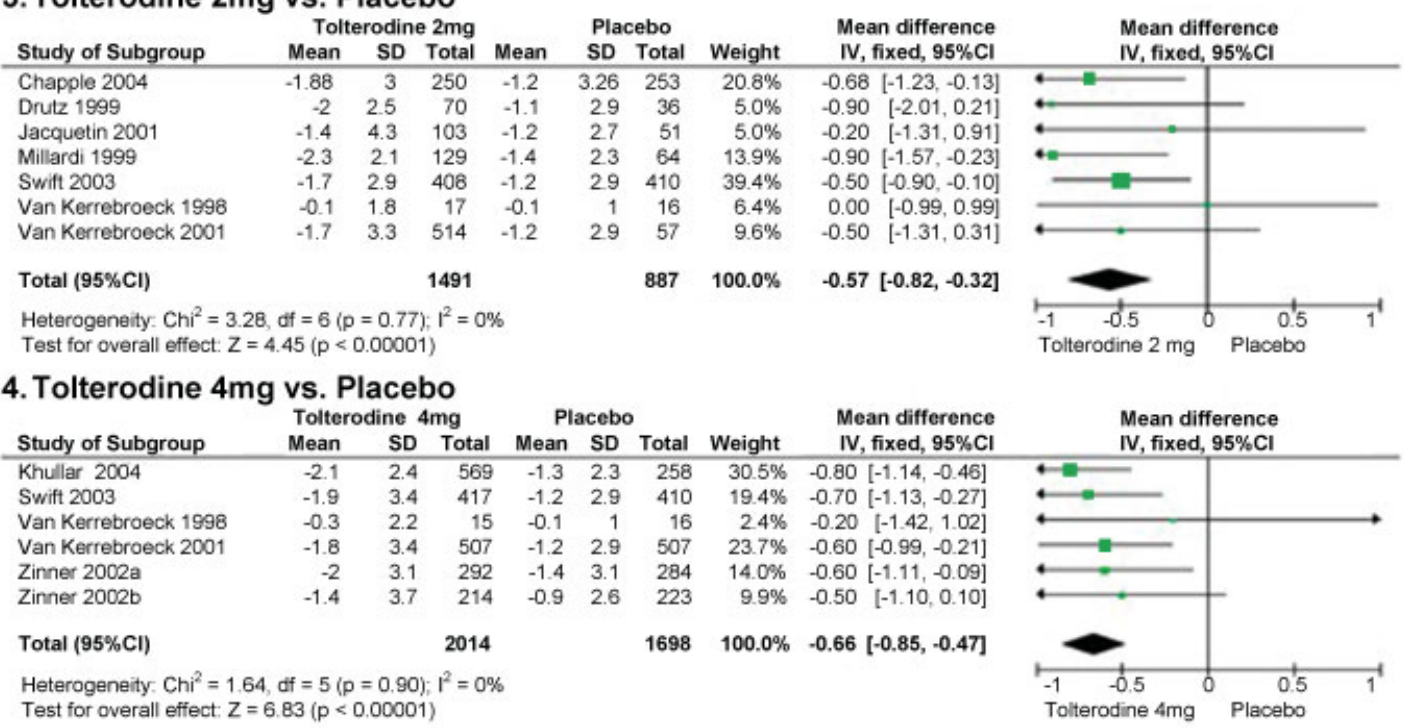

\section{Tolterodine $2 \mathrm{mg}$ vs. Tolterodine $1 \mathrm{mg}$}

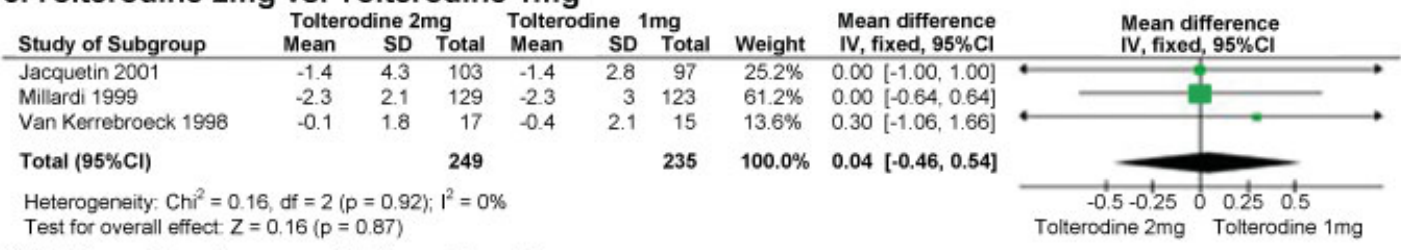

\section{Tolterodine $4 \mathrm{mg}$ vs. Tolterodine $2 \mathrm{mg}$}

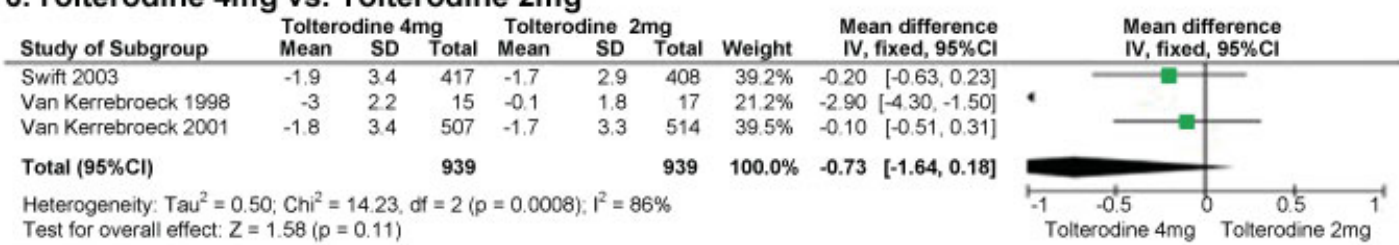

\section{Solifenacin vs. Placebo}

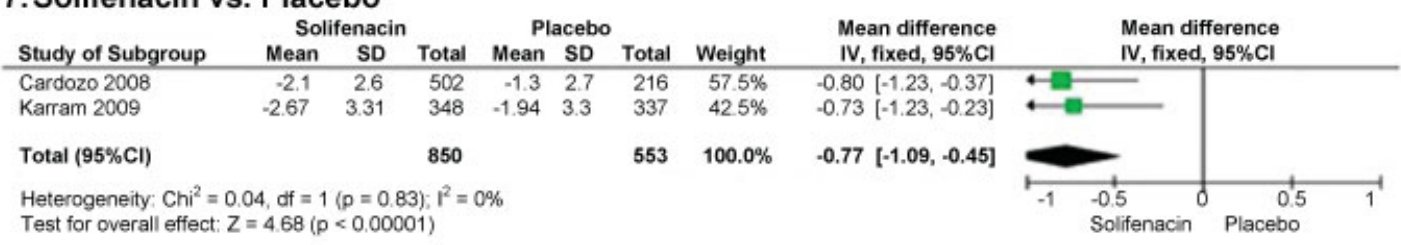

Fig. 3 Forest plot - mean difference in decrease in the number of micturitions per day. 
570 Effectiveness of Anticholinergic Therapy for Overactive Bladders Fonseca et al.

\section{Oxybutynin vs. Tolterodine}

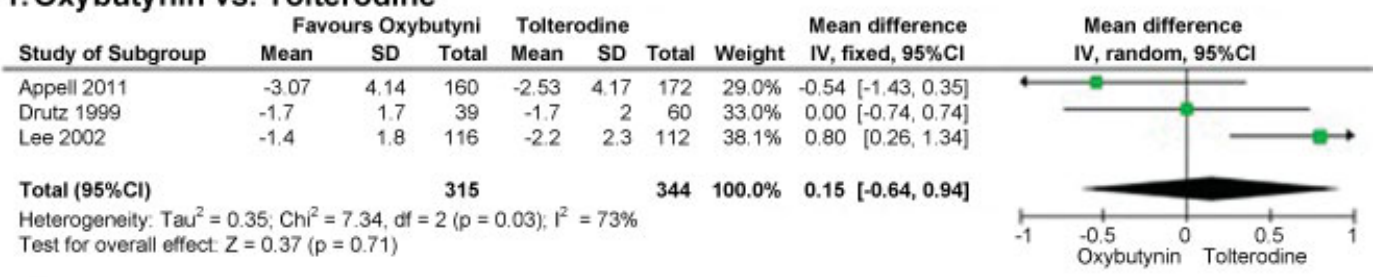

2. Tolterodine $1 \mathrm{mg}$ vs. Placebo

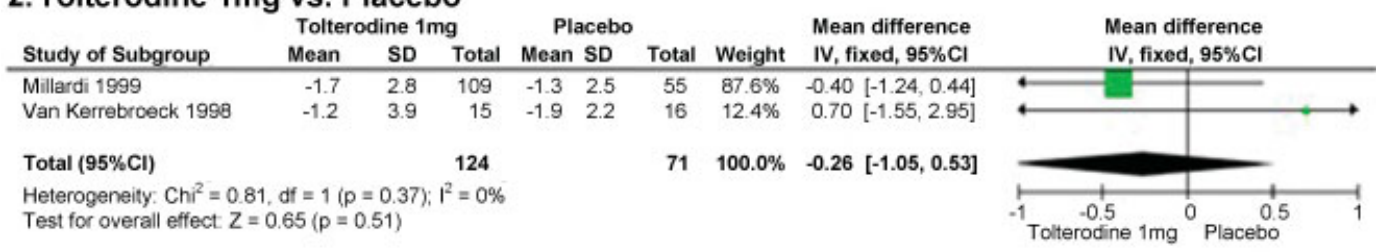

3. Tolterodine $2 \mathrm{mg}$ vs. Placebo

\begin{tabular}{|c|c|c|c|c|c|c|c|c|c|c|}
\hline Study of Subgroup & \multicolumn{3}{|c|}{ Tolterodine $\mathbf{2} \mathbf{m g}$} & \multicolumn{3}{|c|}{ Placebo } & Weight & Mean difference & \multicolumn{2}{|c|}{$\begin{array}{l}\text { Mean difference } \\
\text { IV, fixed, } 95 \% \mathrm{Cl}\end{array}$} \\
\hline Chapple 2004 & -1.14 & 2.15 & 157 & -0.76 & 2.26 & 153 & $40.0 \%$ & $-0.38[-0.87,0.11]$ & & - \\
\hline Drutz 1999 & -1.7 & 2 & 39 & -1 & 2.2 & 33 & $10.1 \%$ & $-0.70[-1.68,0.28]$ & & \\
\hline Milardi 1999 & -1.7 & 2.5 & 117 & -1.3 & 2.5 & 55 & $15.0 \%$ & $-0.40[-1.20,0.40]$ & 4 & \\
\hline Swift 2003 & -1.44 & 5.89 & 408 & -1.03 & 5.74 & 410 & $15.2 \%$ & $-0.41[-1.21,0.39]$ & 4 & \\
\hline Van Kerrebroeck 1998 & -2.4 & 3.5 & 17 & -1.9 & 2.2 & 16 & $2.5 \%$ & $-0.50[-2.48,1.48]$ & 4 & \\
\hline Van Kerrebroeck 2001 & -1.51 & 6.38 & 514 & -0.99 & 5.81 & 507 & $17.2 \%$ & $-0.52[-1.27,0.23]$ & & \\
\hline Total $(95 \% \mathrm{Cl})$ & & & 1252 & & & 1174 & $100.0 \%$ & $-045[-0.76,-0.14]$ & & \\
\hline $\begin{array}{l}\text { Heterogeneity: Chi }{ }^{2}=0 \\
\text { Test for overall effect } Z\end{array}$ & $\begin{array}{l}\mathrm{df}=5(\mathrm{p} \\
82(\mathrm{p}=\end{array}$ & $\begin{array}{l}=1.00 \\
0.005)\end{array}$ & b); $1^{2}=c$ & & & & & & -1 Tolterodine $2 \mathrm{mg}$ & $\begin{array}{r}0.5 \\
\text { Placebc }\end{array}$ \\
\hline
\end{tabular}

\section{Tolterodine $4 \mathrm{mg}$ vs. Placebo}

\begin{tabular}{|c|c|c|c|c|c|c|c|c|c|c|}
\hline \multirow[b]{2}{*}{ Study of Subgroup } & \multicolumn{3}{|c|}{ Tolterodine $4 \mathrm{mg}$} & \multicolumn{3}{|c|}{ Placebo } & \multirow[b]{2}{*}{ Weight } & \multirow{2}{*}{$\begin{array}{l}\text { Mean difference } \\
\text { IV, fixed, } 95 \% \mathrm{Cl}\end{array}$} & \multirow{2}{*}{\multicolumn{2}{|c|}{$\begin{array}{l}\text { Mean difference } \\
\text { IV, fixed, } 95 \% \mathrm{Cl}\end{array}$}} \\
\hline & Mean & SD & Total & Mean & SD & Total & & & & \\
\hline Khullar 2004 & -0.68 & 4.72 & 569 & -0.57 & 5.21 & 258 & $25.6 \%$ & $-0.11[-0.85,0.63]$ & & \\
\hline Swift 2003 & -1.69 & 6.79 & 417 & -1.03 & 5.74 & 410 & $19.4 \%$ & $-0.66[-1.52,0.20]$ & & \\
\hline Van Kerrebroeck 1998 & -1.5 & 1.7 & 15 & -1.9 & 2.2 & 16 & $7.5 \%$ & $0.40[-0.98,1.78]$ & & \\
\hline Van Kerrebroeck 2001 & -1.69 & 6.72 & 507 & -0.99 & 5.81 & 507 & $23.8 \%$ & $-0.70[-1.47,0.07]$ & $\leftarrow$ & \\
\hline Zinner 2002a & -1.71 & 6.64 & 292 & -1.06 & 5.89 & 284 & $13.6 \%$ & $-0.65[-1.67,0.37]$ & & \\
\hline Zinner 2002b & -1.64 & 6.87 & 214 & -0.9 & 5.66 & 223 & $10.2 \%$ & $-0.74[-1.92,0.44]$ & & \\
\hline Total $(95 \% \mathrm{Cl})$ & & & 2014 & & & 1698 & $100.0 \%$ & $-0.46[-0.83,0.08]$ & & \\
\hline $\begin{array}{l}\text { Heterogeneity: } \mathrm{Chi}^{2}=3 \\
\text { Test for overall effect } Z\end{array}$ & $\begin{array}{l}\text { if }=5 \\
37(p\end{array}$ & $\begin{array}{l}0.66) \\
02)\end{array}$ & $1^{2}=0 \%$ & & & & & & ${ }^{-1}$ Tolterod & Pla \\
\hline
\end{tabular}

\section{Tolterodine $2 \mathrm{mg}$ vs. Tolterodine $1 \mathrm{mg}$}

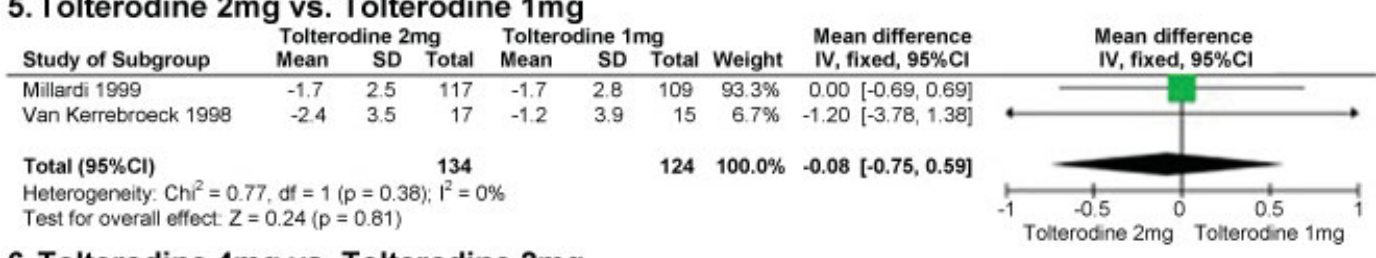

\section{Tolterodine $4 \mathrm{mg}$ vs. Tolterodine $2 \mathrm{mg}$}

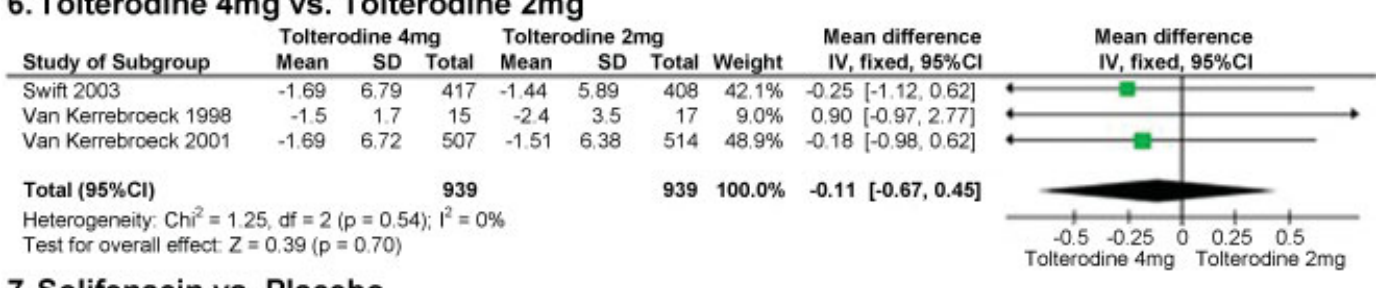

\section{Solifenacin vs. Placebo}

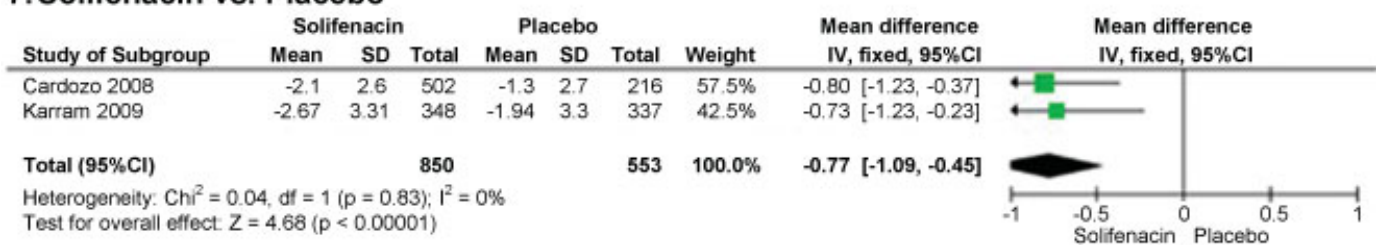

Fig. 4 Forest plot - mean difference in decrease of total incontinence episodes per day. 
1. Oxybutynin vs. Tolterodine

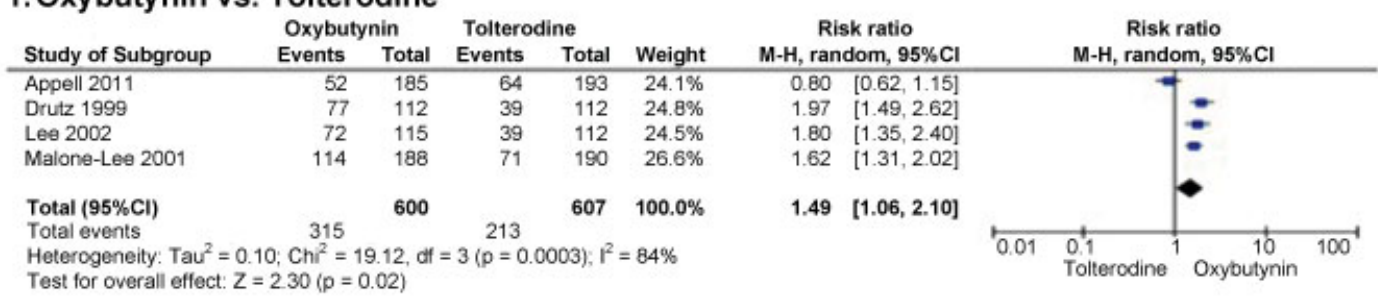

2. Tolterodine $1 \mathrm{mg}$ vs. Placebo

\begin{tabular}{|c|c|c|c|c|c|c|c|c|}
\hline Study of Subgroup & $\begin{array}{l}\text { Tolterodine } \\
\text { Events }\end{array}$ & $\underset{\text { Total }}{1 \mathrm{mg}}$ & $\begin{array}{l}\text { Placebo } \\
\text { Events }\end{array}$ & Total & Weight & $\begin{array}{c}\text { Risk ratio } \\
M \cdot \mathrm{H} \text {, fixed, } 95 \% \mathrm{Cl}\end{array}$ & $\begin{array}{r}\text { Risk } \\
M-H \text {, fixe }\end{array}$ & $\begin{array}{l}\text { ratio } \\
\text { d, } 95 \% \mathrm{Cl}\end{array}$ \\
\hline Jacquetin 2001 & 20 & 97 & 3 & 51 & $27.2 \%$ & $3.51[1.09,11.24]$ & & \\
\hline Millardi 1999 & 29 & 123 & 8 & 64 & $72.8 \%$ & $1.89[0.92,3.88]$ & & \\
\hline Total $(95 \% \mathrm{Cl})$ & & 220 & & 115 & $100.0 \%$ & $2.33[1.26,4.29]$ & & \\
\hline $\begin{array}{l}\text { Total events } \\
\text { Heterogeneity: Chi }{ }^{2}\end{array}$ & $\begin{array}{c}49 \\
\text { df }=1(p=\end{array}$ & 7): $1^{2}=$ & 11 & & & & $0.01 \quad 0.19$ & \begin{tabular}{|c|c|}
10 & 100 \\
Tolterodine $1 \mathrm{mg}$
\end{tabular} \\
\hline
\end{tabular}

Test for overall effect: $Z=2.70(p=0.007)$

\section{Tolterodine $\mathbf{2 m g}$ vs. Placebo}

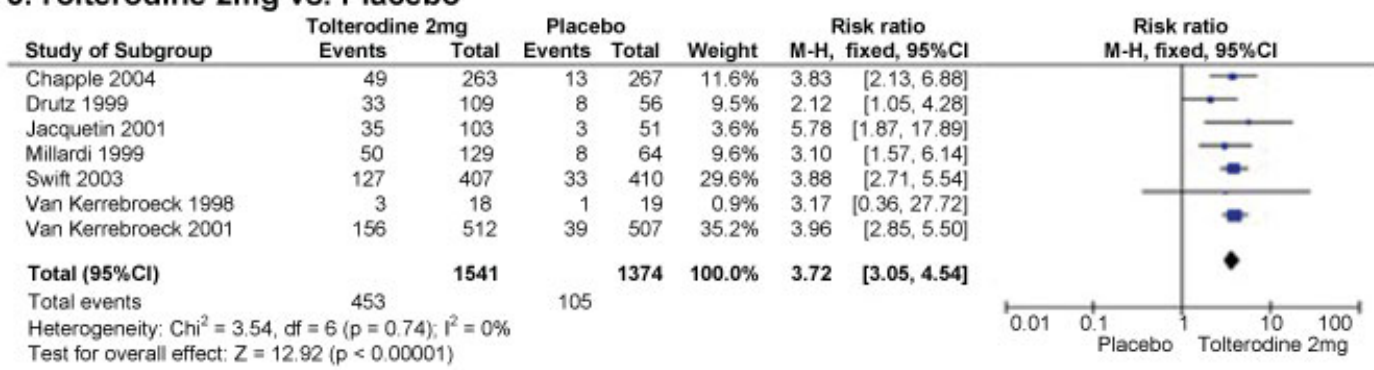

\section{Tolterodine $4 \mathrm{mg}$ vs. Placebo}

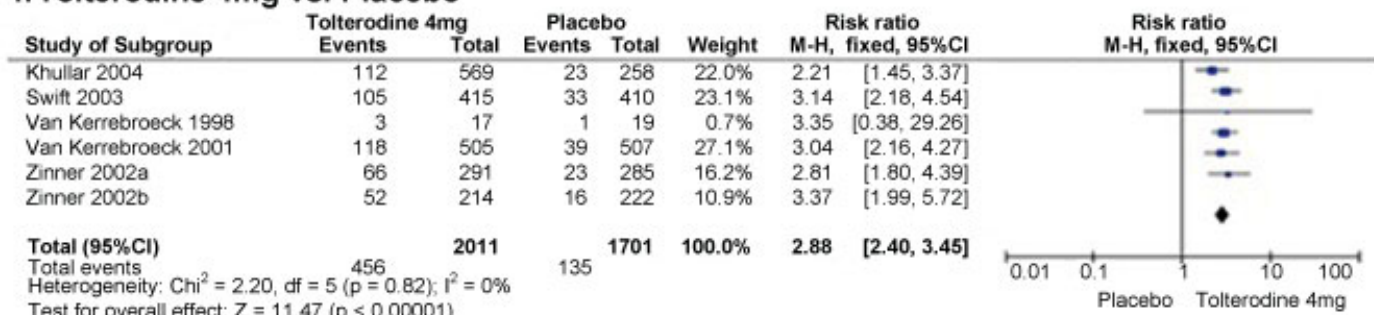

\section{Tolterodine $2 \mathrm{mg}$ vs. Tolterodine $1 \mathrm{mg}$}

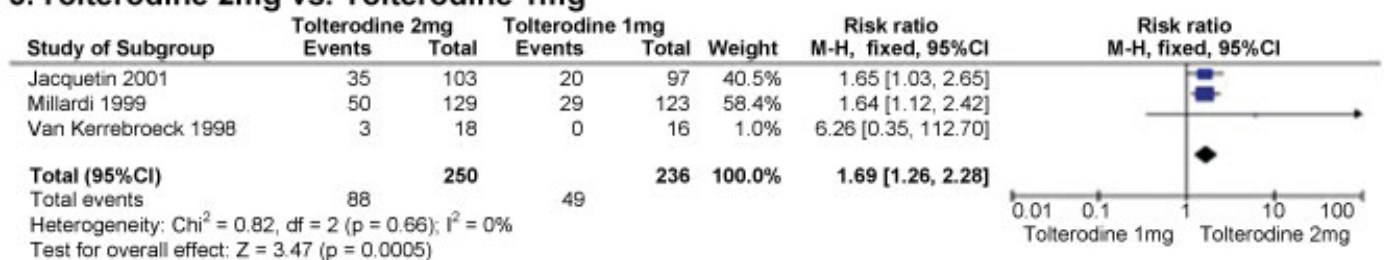

\section{Tolterodine $4 \mathrm{mg}$ vs. Tolterodine $2 \mathrm{mg}$}

\begin{tabular}{|c|c|c|c|c|c|c|c|c|c|}
\hline \multirow{2}{*}{$\begin{array}{l}\text { Study of Subgroup } \\
\text { Swift } 2003\end{array}$} & \multicolumn{2}{|c|}{$\begin{array}{l}\text { Tolterodine 4mg } \\
\text { Events Total }\end{array}$} & $\begin{array}{l}\text { Tolterodine } \\
\text { Events }\end{array}$ & Total & \multirow{2}{*}{ Weight } & \multirow{2}{*}{$\begin{array}{c}\begin{array}{c}\text { Risk ratio } \\
\text { M-H, fixed, 95\% Cl }\end{array} \\
0.81[0.65,1.01]\end{array}$} & \multicolumn{3}{|c|}{$\begin{array}{c}\text { Risk ratio } \\
\mathrm{M}-\mathrm{H} \text {, fixed, } 95 \% \mathrm{Cl}\end{array}$} \\
\hline & 105 & 415 & 127 & 407 & & & $\rightarrow$ & & \\
\hline Van Kerrebroeck 1998 & 3 & 17 & 3 & 18 & $1.0 \%$ & $1.06[0.25,4.54]$ & & & \\
\hline Van Kerrebroeck 2001 & 118 & 505 & 156 & 512 & $54.2 \%$ & $0.77[0.62,0.94]$ & 붕 & & \\
\hline Total $(95 \% \mathrm{Cl})$ & & 937 & & 937 & $100.0 \%$ & $0.79[0.68,0.92]$ & & & \\
\hline $\begin{array}{l}\text { Total events } \\
\text { Heterogeneity: } \text { Chi }^{2}=0.2 \\
\text { Test for overall effect: } Z\end{array}$ & $\begin{array}{c}226 \\
d f=2(p=0 \\
311(p=0.0)\end{array}$ & $1^{2}=$ & 286 & & & & $\begin{array}{lll}0.1 & 0.2 & 0.5 \\
0.1\end{array}$ & $\begin{array}{c}1 \\
2 \\
\text { Tolterodir }\end{array}$ & 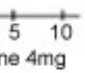 \\
\hline
\end{tabular}

\section{Solifenacin vs. Placebo}

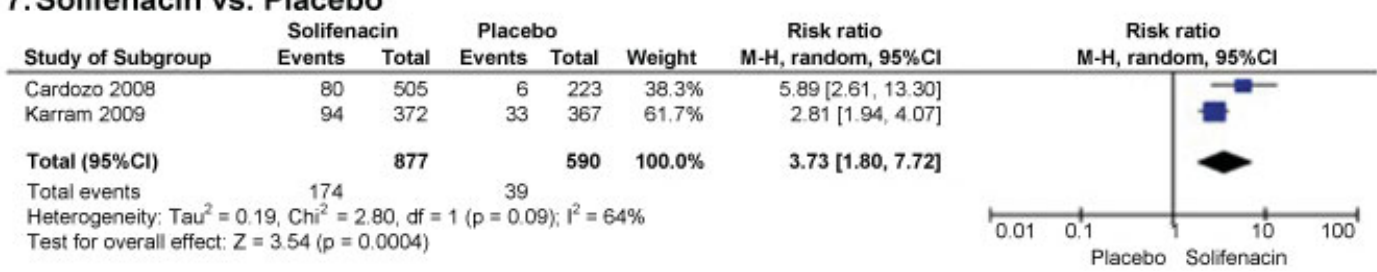

Fig. 5 Forest plot - Risk Ratio (RR) of dry mouth. 


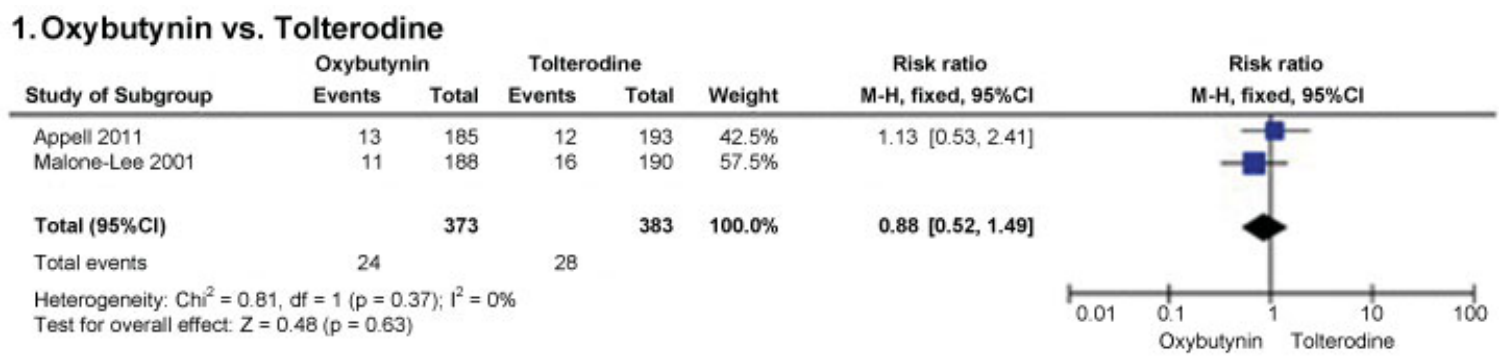

\section{Tolterodine $2 \mathrm{mg}$ vs. Placebo}

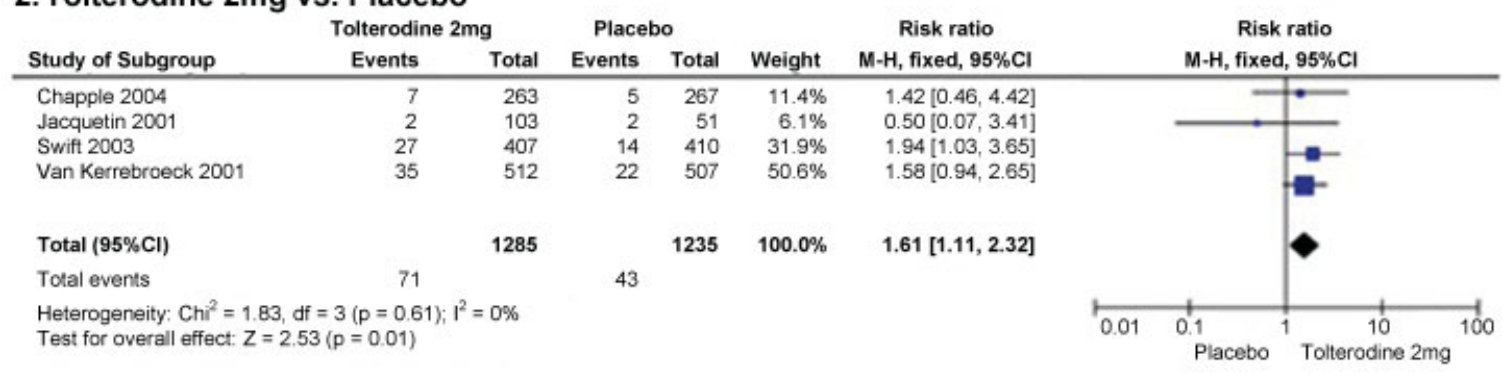

\section{Tolterodine $4 \mathrm{mg}$ vs. Placebo}

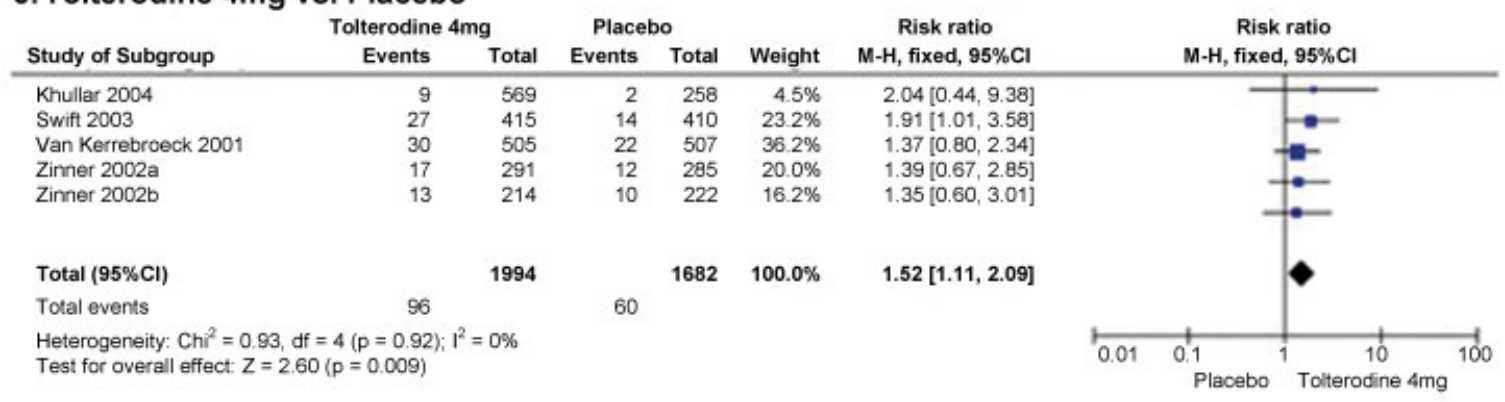

\section{Solifenacin vs. Placebo}

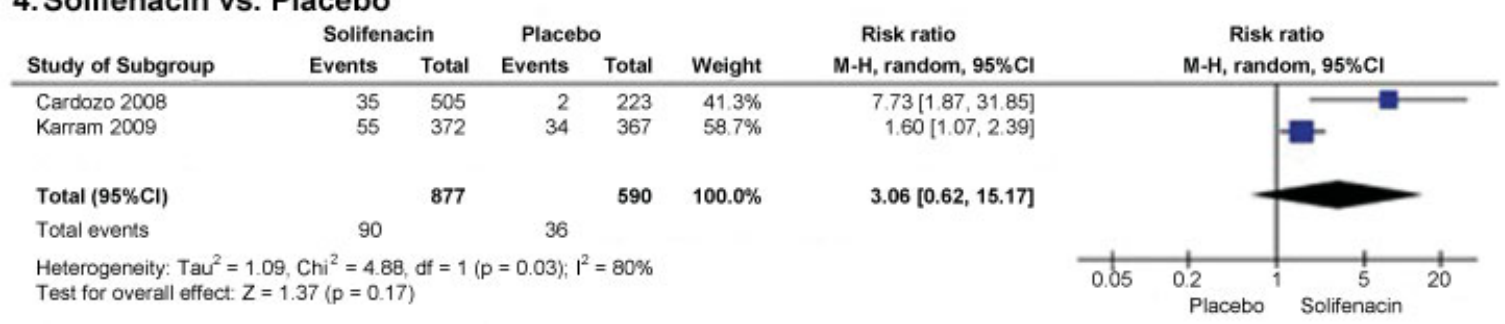

Fig. 6 Forest plot - Risk Ratio (RR) of constipation.

This systematic review showed that there is no significant difference in the mean decrease in UUI episodes per day between oxybutynin and tolterodine. Although there was a trend of a higher reduction in UUI episodes with the use of oxybutynin, the difference was not statistically significant. It was not possible to perform comparisons between oxybutynin versus solifenacin, oxybutynin versus darifenacin, tolterodine versus solifenacin, tolterodine versus darifenacin, and solifenacin versus darifenacin due to limitations in data reporting (that is, studies without a measure of variation) and the lack of similarity in measures.

Regarding the decrease in the number of micturitions per day, which was another important primary outcome, the results favored tolterodine in its various dosages and solifenacin when compared with placebo. The comparison between oxybutynin and tolterodine showed no significant difference in treatment efficacy across any of the outcomes; the same was found for the comparisons of tolterodine in its various dosages. As result of the relative paucity of data that qualified for inclusion in the meta-analysis - and that directly compared pharmacological agents -, it is impossible to report definitively whether any specific agent is superior to another in terms of efficacy.

Antimuscarinic agents may be associated with adverse effects. The human bladder tissue contains M2 and M3 muscarinic receptors. The $\mathrm{M} 3$ subtype has been identified as the primary mediator of detrusor contraction in response to cholinergic activation. ${ }^{52,53}$ Different subtypes of muscarinic receptors are widely distributed in the body. M1 receptors in the brain and salivary glands are involved in cognition and in the 
production of mucous saliva; ${ }^{54,55} \mathrm{M} 2$ receptors in the cardiovascular system play a role in mediating heart rate and cardiac output; $^{56}$ and M5 receptors in the eye are involved in ciliary muscle contraction. ${ }^{57-59}$ As a result, antimuscarinic agents, which bind to some or all of these receptors, are effective in treating OAB symptoms, but they may also be associated with adverse effects such as dry mouth, constipation, cognitive impairment, tachycardia, and blurred vision. ${ }^{57}$ This systematic review showed that oxybutynin was associated with significantly higher rates of dry mouth when compared with tolterodine. When compared with placebo, tolterodine, in its various dosages, and solifenacin were associated with significantly higher rates of dry mouth. The group of patients that used tolterodine $4 \mathrm{mg}$ presented lower risk when compared with the group treated with tolterodine $2 \mathrm{mg}$. This can be explained by the fact that tolterodine $4 \mathrm{mg}$ is an extended-release (ER) presentation. Compared with the immediate-release drug, tolterodine ER releases the drug in a steady and constant manner, thus lowering peaks. This translates into more constant serum concentrations and theoretically improves patient tolerability. ${ }^{60}$ Concerning constipation, differences were not found between oxybutynin and tolterodine. Significantly high rates of constipation were found in patients treated with tolterodine $2 \mathrm{mg}$ and $4 \mathrm{mg}$ when compared with placebo.

The current data demonstrate that a substantial proportion of patients discontinue anticholinergic drugs, with $75-90 \%$ of patients discontinuing therapy within 12 months. Among those studies that provided information about the reasons for the discontinuation of the therapy, the most frequently cited reasons were that the medication did not work as expected, and that the medication's side effects were not desirable. ${ }^{7}$ We did not find a statistical difference associated with withdrawals resulting from drug-related adverse effects.

New drugs for the treatment of OAB are emerging, such as imidafenacin and tarafenacin, but they are not available in Brazil yet. Mirabegron, a $\beta 3$-adrenoreceptor agonist, has just recently been released into the Brazilian market with some promising results, especially when associated with regular antimuscarinic drugs. ${ }^{61,62}$

The quality of the available evidence that supports these results is moderate. The main limitation of the available evidence concerning $\mathrm{OAB}$ treatment is that although there is a large amount of RCTs, it is not possible to combine all of the data in a meta-analysis due to their heterogeneity. If the goal of a meta-analysis is to estimate the MD between two treatments, then the means, sample sizes, and a measure of variation (standard deviation, standard error, or a confidence interval) are required. Thus, many of the available RCTs on $\mathrm{OAB}$ treatment did not contribute to the meta-analysis, and were excluded from our study. Unfortunately, we discovered a lack of high-quality evidence pertaining to the available drugs and dosages for the treatment of OAB in Brazil that can inform clinical decision making for patients and care providers.

In summary, the results of this meta-analysis suggest that there is a moderate to high quality of evidence supporting the benefits of using anticholinergic drugs in alleviating $O A B$ symptoms when compared with placebo. Despite its lower improvement in primary and secondary outcomes when compared with anticholinergics, the use of placebo contributed to many of the improvements in OAB symptoms. It is still not clear if any one specific drug available in Brazil has any advantage over the others. The use of these drugs is associated with adverse effects (mainly dry mouth and constipation), although the use of these agents is not related to an increase in the number of withdrawals.

\section{References}

1 Haylen BT, de Ridder D, Freeman RM, et al; International Urogynecological Association; International Continence Society. An International Urogynecological Association (IUGA)/International Continence Society (ICS) joint report on the terminology for female pelvic floor dysfunction. Neurourol Urodyn 2010;29(1): 4-20

2 Irwin DE, Milsom I, Hunskaar S, et al. Population-based survey of urinary incontinence, overactive bladder, and other lower urinary tract symptoms in five countries: results of the EPIC study. Eur Urol 2006;50(6):1306-1314, discussion 1314-1315

3 Stewart WF, Van Rooyen JB, Cundiff GW, et al. Prevalence and burden of overactive bladder in the United States. World J Urol 2003;20(6):327-336

4 Townsend MK, Minassian VA, Okereke OI, Resnick NM, Grodstein F. Urinary incontinence and prevalence of high depressive symptoms in older black versus white women. Int Urogynecol J Pelvic Floor Dysfunct 2014;25(6):823-829

5 Sexton CC, Notte SM, Maroulis C, et al. Persistence and adherence in the treatment of overactive bladder syndrome with anticholinergic therapy: a systematic review of the literature. Int J Clin Pract 2011;65(5):567-585

6 Moroni RM, Magnani PS, Haddad JM, Castro RdeA, Brito LG. Conservative treatment of stress urinary incontinence: a systematic review with meta-analysis of randomized controlled trials. Rev Bras Ginecol Obstet 2016;38(2):97-111

7 Gormley EA, Lightner DJ, Burgio KL, et al; American Urological Association; Society of Urodynamics, Female Pelvic Medicine \& Urogenital Reconstruction. Diagnosis and treatment of overactive bladder (non-neurogenic) in adults: AUA/SUFU guideline. J Urol 2012;188(6, Suppl)2455-2463

8 Thiagamoorthy G, Cardozo L, Robinson D. Current and future pharmacotherapy for treating overactive bladder. Expert Opin Pharmacother 2016;17(10):1317-1325

9 Brown ET, Martin L, Dmochowski RR. New evidence in the treatment of overactive bladder. Curr Opin Obstet Gynecol 2015;27(5):366-372

10 Lawrence M, Guay DR, Benson SR, Anderson MJ. Immediaterelease oxybutynin versus tolterodine in detrusor overactivity: a population analysis. Pharmacotherapy 2000;20(4): 470-475

11 Juliato CR, Santos Júnior LC, Haddad JM, Castro RA, Lima M, Castro EB. Mesh surgery for anterior vaginal wall prolapse: a metaanalysis. Rev Bras Ginecol Obstet 2016;38(7):356-364

12 Moher D, Liberati A, Tetzlaff J, Altman DG; PRISMA Group. Preferred reporting items for systematic reviews and meta-analyses: the PRISMA statement. BMJ 2009;339:b2535

13 Higgins JP, Green S. Cochrane handbook for systematic reviews of interventions. Version 5.1.0. London: The Cochrane Colaboration; 2011

14 Higgins JP, Thompson SG, Deeks JJ, Altman DG. Measuring inconsistency in meta-analyses. BMJ 2003;327(7414):557-560

15 Appell RA, Sand P, Dmochowski R, et al; Overactive Bladder: Judging Effective Control and Treatment Study Group. Prospective randomized controlled trial of extended-release oxybutynin chloride and tolterodine tartrate in the treatment of overactive 
bladder: results of the OBJECT Study. Mayo Clin Proc 2001;76(4): 358-363

16 Drutz HP, Appell RA, Gleason D, Klimberg I, Radomski S. Clinical efficacy and safety of tolterodine compared to oxybutynin and placebo in patients with overactive bladder. Int Urogynecol J Pelvic Floor Dysfunct 1999;10(5):283-289

17 Lee JG, Hong JY, Choo MS, et al. Tolterodine: as effective but better tolerated than oxybutynin in Asian patients with symptoms of overactive bladder. Int J Urol 2002;9(5):247-252

18 Malone-Lee J, Shaffu B, Anand C, Powell C. Tolterodine: superior tolerability than and comparable efficacy to oxybutynin in individuals 50 years old or older with overactive bladder: a randomized controlled trial. J Urol 2001;165(5):1452-1456

19 Chapple CR, Rechberger T, Al-Shukri S, et al; YM-905 Study Group. Randomized, double-blind placebo- and tolterodine-controlled trial of the once-daily antimuscarinic agent solifenacin in patients with symptomatic overactive bladder. BJU Int 2004;93(3): 303-310

20 Jacquetin B, Wyndaele J. Tolterodine reduces the number of urge incontinence episodes in patients with an overactive bladder. Eur J Obstet Gynecol Reprod Biol 2001;98(1):97-102

21 Khullar V, Hill S, Laval KU, Schiøtz HA, Jonas U, Versi E. Treatment of urge-predominant mixed urinary incontinence with tolterodine extended release: a randomized, placebo-controlled trial. Urology 2004;64(2):269-274, discussion 274-275

22 Millard R, Tuttle J, Moore K, et al. Clinical efficacy and safety of tolterodine compared to placebo in detrusor overactivity. J Urol 1999;161(5):1551-1555

23 Swift S, Garely A, Dimpfl T, Payne C; Tolterodine Study Group. A new once-daily formulation of tolterodine provides superior efficacy and is well tolerated in women with overactive bladder. Int Urogynecol J Pelvic Floor Dysfunct 2003;14(1):50-54, discussion 54-55

24 Van Kerrebroeck PE, Amarenco G, Thüroff JW, et al. Dose-ranging study of tolterodine in patients with detrusor hyperreflexia. Neurourol Urodyn 1998;17(5):499-512

25 Van Kerrebroeck P, Kreder K, Jonas U, Zinner N, Wein A; Tolterodine Study Group. Tolterodine once-daily: superior efficacy and tolerability in the treatment of the overactive bladder. Urology 2001;57(3):414-421

26 Zinner NR, Mattiasson A, Stanton SL. Efficacy, safety, and tolerability of extended-release once-daily tolterodine treatment for overactive bladder in older versus younger patients. J Am Geriatr Soc 2002;50(5):799-807

27 Cardozo L, Hessdörfer E, Milani R, et al; SUNRISE Study Group. Solifenacin in the treatment of urgency and other symptoms of overactive bladder: results from a randomized, double-blind, placebo-controlled, rising-dose trial. BJU Int 2008;102(9): 1120-1127

28 Karram MM, Toglia MR, Serels SR, Andoh M, Fakhoury A, ForeroSchwanhaeuser $\mathrm{S}$. Treatment with solifenacin increases warning time and improves symptoms of overactive bladder: results from VENUS, a randomized, double-blind, placebo-controlled trial Urology 2009;73(1):14-18

29 But I, Goldstajn MS, Oresković S. Comparison of two selective muscarinic receptor antagonists (solifenacin and darifenacin) in women with overactive bladder-the SOLIDAR study. Coll Antropol 2012;36(4):1347-1353

30 Armstrong RB, Dmochowski RR, Sand PK, Macdiarmid S. Safety and tolerability of extended-release oxybutynin once daily in urinary incontinence: combined results from two phase 4 controlled clinical trials. Int Urol Nephrol 2007;39(4): 1069-1077

31 Barkin J, Corcos J, Radomski S, et al; UROMAX Study Group. A randomized, double-blind, parallel-group comparison of controlled- and immediate-release oxybutynin chloride in urge urinary incontinence. Clin Ther 2004;26(7):1026-1036
32 Leung HY, Yip SK, Cheon C, et al. A randomized controlled trial of tolterodine and oxybutynin on tolerability and clinical efficacy for treating Chinese women with an overactive bladder. BJU Int 2002; 90(4):375-380

33 Jonas U, Höfner K, Madersbacher H, Holmdahl TH; The International Study Group. Efficacy and safety of two doses of tolterodine versus placebo in patients with detrusor overactivity and symptoms of frequency, urge incontinence, and urgency: urodynamic evaluation. World J Urol 1997;15(2):144-151

34 Wagg A, Dale M, Tretter R, Stow B, Compion G. Randomised multicentre, placebo-controlled, double-blind crossover study investigating the effect of solifenacin and oxybutynin in elderly people with mild cognitive impairment: the SENIOR study. Eur Urol 2013;64(1):74-81

35 Abrams P, Kelleher C, Huels J, Quebe-Fehling E, Omar MA, Steel M. Clinical relevance of health-related quality of life outcomes with darifenacin. BJU Int 2008;102(2):208-213

36 Diokno AC, Appell RA, Sand PK, et al; OPERA Study Group. Prospective, randomized, double-blind study of the efficacy and tolerability of the extended-release formulations of oxybutynin and tolterodine for overactive bladder: results of the OPERA trial. Mayo Clin Proc 2003;78(6):687-695

37 Lackner TE, Wyman JF, McCarthy TC, Monigold M, Davey C. Efficacy of oral extended-release oxybutynin in cognitively impaired older nursing home residents with urge urinary incontinence: a randomized placebo-controlled trial. J Am Med Dir Assoc 2011;12(9):639-647

38 Sand PK, Miklos J, Ritter H, Appell R. A comparison of extendedrelease oxybutynin and tolterodine for treatment of overactive bladder in women. Int Urogynecol J Pelvic Floor Dysfunct 2004; 15(4):243-248

39 Versi E, Appell R, Mobley D, Patton W, Saltzstein D; The Ditropan XL Study Group. Dry mouth with conventional and controlledrelease oxybutynin in urinary incontinence. Obstet Gynecol 2000; 95(5):718-721

40 Yoo DS, Han JY, Lee KS, Choo MS. Prescription pattern of oxybutynin ER in patients with overactive bladder in real life practice: a multicentre, open-label, prospective observational study. Int J Clin Pract 2012;66(2):132-138

41 Chapple CR, Fianu-Jonsson A, Indig M, et al; STAR study group. Treatment outcomes in the STAR study: a subanalysis of solifenacin $5 \mathrm{mg}$ and tolterodine ER $4 \mathrm{mg}$. Eur Urol 2007;52(4): 1195-1203

42 Dmochowski R, Abrams P, Marschall-Kehrel D, Wang JT, Guan Z. Efficacy and tolerability of tolterodine extended release in male and female patients with overactive bladder. Eur Urol 2007;51(4): 1054-1064, discussion 1064

43 Rackley R, Weiss JP, Rovner ES, Wang JT, Guan Z; 037 STUDY GROUP. Nighttime dosing with tolterodine reduces overactive bladder-related nocturnal micturitions in patients with overactive bladder and nocturia. Urology 2006;67(4):731-736, discussion 736

44 Rentzhog L, Stanton SL, Cardozo L, Nelson E, Fall M, Abrams P. Efficacy and safety of tolterodine in patients with detrusor instability: a dose-ranging study. Br J Urol 1998;81(1):42-48

45 Vardy MD, Mitcheson HD, Samuels TA, et al. Effects of solifenacin on overactive bladder symptoms, symptom bother and other patient-reported outcomes: results from VIBRANT - a doubleblind, placebo-controlled trial. Int J Clin Pract 2009;63(12): 1702-1714

46 Haab F, Stewart L, Dwyer P. Darifenacin, an M3 selective receptor antagonist, is an effective and well-tolerated once-daily treatment for overactive bladder. Eur Urol 2004;45(4):420-429, discussion 429

47 Hill S, Khullar V, Wyndaele JJ, Lheritier K; Darifenacin Study Group. Dose response with darifenacin, a novel once-daily M3 selective receptor antagonist for the treatment of overactive 
bladder: results of a fixed dose study. Int Urogynecol J Pelvic Floor Dysfunct 2006;17(3):239-247

48 Khullar V, Foote J, Seifu Y, Egermark M. Time-to-effect with darifenacin in overactive bladder: a pooled analysis. Int Urogynecol J Pelvic Floor Dysfunct 2011;22(12):1573-1580

49 Steers W, Corcos J, Foote J, Kralidis G. An investigation of dose titration with darifenacin, an M3-selective receptor antagonist. BJU Int 2005;95(4):580-586

50 Zinner N, Tuttle J, Marks L. Efficacy and tolerability of darifenacin, a muscarinic M3 selective receptor antagonist (M3 SRA), compared with oxybutynin in the treatment of patients with overactive bladder. World J Urol 2005;23(4):248-252

51 Zinner N, Susset J, Gittelman M, Arguinzoniz M, Rekeda L, Haab F. Efficacy, tolerability and safety of darifenacin, an $\mathrm{M}(3)$ selective receptor antagonist: an investigation of warning time in patients with OAB. Int J Clin Pract 2006;60(1):119-126

52 Fetscher C, Fleichman M, Schmidt M, Krege S, Michel MC. M(3) muscarinic receptors mediate contraction of human urinary bladder. Br J Pharmacol 2002;136(5):641-643

53 Chess-Williams R. Muscarinic receptors of the urinary bladder: detrusor, urothelial and prejunctional. Auton Autacoid Pharmacol 2002;22(3):133-145

54 Fisher A, Michaelson DM, Brandeis R, Haring R, Chapman S, Pittel Z. M1 muscarinic agonists as potential disease-modifying agents in Alzheimer's disease. Rationale and perspectives. Ann N Y Acad Sci 2000;920:315-320
55 Culp DJ, Luo W, Richardson LA, Watson GE, Latchney LR. Both M1 and M3 receptors regulate exocrine secretion by mucous acini. Am J Physiol 1996;271(6 Pt 1):C1963-C1972

56 Bymaster FP, Carter PA, Zhang L, et al. Investigations into the physiological role of muscarinic M2 and M4 muscarinic and M4 receptor subtypes using receptor knockout mice. Life Sci 2001; 68(22-23):2473-2479

57 Andersson KE. Potential benefits of muscarinic M3 receptor sensitivity. Eur Urol Suppl 2002;1(4):23-28

58 Gil DW, Krauss HA, Bogardus AM, WoldeMussie E. Muscarinic receptor subtypes in human iris-ciliary body measured by immunoprecipitation. Invest Ophthalmol Vis Sci 1997;38(7): 1434-1442

59 Choppin A, Eglen RM. Pharmacological characterization of muscarinic receptors in dog isolated ciliary and urinary bladder smooth muscle. Br J Pharmacol 2001;132(4):835-842

60 Chung DE, Te AE. Tolterodine extended-release for overactive bladder. Expert Opin Pharmacother 2009;10(13):2181-2194

61 Karmarkar R, Khullar V. Emerging drugs for overactive bladder. Expert Opin Emerg Drugs 2015;20(4):613-624

62 Drake MJ, Chapple C, Esen AA, et al; BESIDE study investigators. Efficacy and safety of mirabegron add-on therapy to solifenacin in incontinent overactive bladder patients with an inadequate response to initial 4-week solifenacin monotherapy: a randomised doubleblind multicentre phase 3b study (BESIDE). Eur Urol 2016;70(1): 136-145 